ARTICLE

\title{
A solvent-assisted ligand exchange approach enables metal-organic frameworks with diverse and complex architectures
}

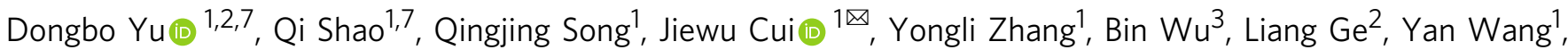
Yong Zhang ${ }^{1}$, Yongqiang Qin ${ }^{1}$, Robert Vajtai (1) ${ }^{4,5}$, Pulickel M. Ajayan (1) ${ }^{4 凶}$, Huanting Wang (i) ${ }^{6}$,

Tongwen $\mathrm{Xu}{ }^{2 \otimes} \&$ Yucheng $\mathrm{Wu}^{1 凶}$

Unlike inorganic crystals, metal-organic frameworks do not have a well-developed nanostructure library, and establishing their appropriately diverse and complex architectures remains a major challenge. Here, we demonstrate a general route to control metal-organic framework structure by a solvent-assisted ligand exchange approach. Thirteen different types of metal-organic framework structures have been prepared successfully. To demonstrate a proof of concept application, we used the obtained metal-organic framework materials as precursors for synthesizing nanoporous carbons and investigated their electrochemical $\mathrm{Na}^{+}$ storage properties. Due to the unique architecture, the one-dimensional nanoporous carbon derived from double-shelled $\mathrm{ZnCo}$ bimetallic zeolitic imidazolate framework nanotubes exhibits high specific capacity as well as superior rate capability and cycling stability. Our study offers an avenue for the controllable preparation of well-designed meta-organic framework structures and their derivatives, which would further broaden the application opportunities of metal-organic framework materials.

\footnotetext{
${ }^{1}$ School of Materials Science and Engineering, Hefei University of Technology, Hefei 230009, P. R. China. ${ }^{2}$ CAS Key Laboratory of Soft Matter Chemistry, Collaborative Innovation Center of Chemistry for Energy Materials, School of Chemistry and Material Science University of Science and Technology of China, Hefei 230026, P. R. China. ${ }^{3}$ Key Laboratory of Environment-Friendly Polymeric Materials of Anhui Province, School of Chemistry \& Chemical Engineering Anhui University, Hefei 230601, P. R. China. ${ }^{4}$ Department of Materials Science and NanoEngineering, Rice University, Houston, TX 77005, USA. ${ }^{5}$ University of Szeged, Interdisciplinary Excellence Centre, Department of Applied and Environmental Chemistry, University of Szeged, Rerrich Béla tér 1.,

Szeged, Hungary. ${ }^{6}$ Department of Chemical Engineering, Monash University, Clayton, VIC 3800, Australia. ${ }^{7}$ These authors contributed equally: Dongbo Yu, Qi Shao.凶email: jwcui@hfut.edu.cn; ajayan@rice.edu; twxu@ustc.edu.cn; ycwu@hfut.edu.cn
} 
$\mathrm{M}$ etal-organic frameworks (MOFs) are a class of porous inorganic-organic hybrid crystals ${ }^{1}$, which have been employed in a wide range of applications, owing to their fascinating properties such as high surface area, structural and functional flexibility ${ }^{2-4}$. The study of MOFs has emerged as one of the most advanced research frontiers. To realize more targeted functionalities, substantial efforts mainly focus on the development of new types of $\mathrm{MOFs}^{5,6}$, molecular level manipulations (e.g., chemical modification and aperture size adjustment) $)^{1,7}$, and megascopic manufacturing (e.g., MOF membranes) ${ }^{8}$. By contrast, only a small number of attempts have been devoted to tailoring MOF architectures on the micro-/nano-scale, even if architectural design is known to have a vital effect on performance ${ }^{9-11}$. Some latest significant progresses actually benefit from very common architectures such as MOF@MOF core-shell structures and MOF hollow structures ${ }^{9-18}$. Nevertheless, unlike inorganic crystals which have abundant nanostructure libraries and well-developed preparation approaches ${ }^{19,20}$, MOFs commonly lack nano-morphological diversity and nanostructural complexity, a large proportion of MOFs are solid particle shapes, which extremely limits the application of MOF materials.

Currently, most of the reported core-shell MOFs are prepared by an epitaxial growth method ${ }^{21}$, and the shell MOFs often need to have the identical topology structure of the core MOFs ${ }^{11,15,16}$. In the light of crystal growth theory, nucleation favors a coherent phase boundary between the substrate and target crystal. To hybridize MOFs with different topology, a surfactant-mediated strategy is proposed ${ }^{17,18}$, while there are still many core MOFs totally uncovered or partially covered by the shell MOFs. Another double hydrophilic block copolymer-modulated method is also demonstrated to design a core-shell MOF hybrid with relatively low crystallinity of MOF shell 22 . In addition, since the convex surface of one-dimensional (1D) MOFs is not as favorable for crystallization as the flat plane of polyhedral MOFs, 1D MOF@MOF core-shell nanostructures have rarely been reported. Different from core-shell structure, hollow structure possesses a specific enclosed space, representing another very important configuration ${ }^{10}$. To date, simple single-shelled hollow particles dominate the majority of hollow MOFs, as some more complex hollow MOFs start to appear in recent reports ${ }^{13,14}$. However, the successful fabrication of these complex structures involves complicated growth-and-etching procedures and has various extreme prerequisites; each of these methods is unlikely to be extended to other MOFs and is solely for a specific MOF. Therefore, given that the study of MOF nanostructure regulation is in its infancy, the researchers have sufficient motivation to develop new, facile and versatile synthesis strategies in order to enrich the MOF nanostructure library.

In this manuscript, we successfully fabricate thirteen different MOF nanoarchitectures via a developed solvent-assisted ligand exchange (SALE) approach ${ }^{23-26}$, although SALE is conventionally used for MOF-related functional modifications. Considering that, in theory, the thermodynamic driving force during the exchange process is the formation of more stable coordination bonds ${ }^{27}$, we thus choose stable zeolitic imidazolate frameworks (ZIFs) as target daughter MOFs, and 1D/2D/3D MOFs with weaker bonds serve as the mother MOFs (Fig. 1). Because a typical SALE transformation generally involves two interweaving steps of dissolution and recrystallization ${ }^{28}$, through precisely adjusting the balance between the cleavage of old bonds and the establishment of new bonds, we can obtain different kinds of 1D/2D/3D MOF nanostructures, as shown in Supplementary Fig. 1. These architectures cover most of the nanostructures in inorganic crystals. Very encouragingly, 1D double-shelled nanotube, nanowire-nanotube and peapod-like MOF structures have been synthesized. It is reported that some MOFs can be directly used for electrochemical applications ${ }^{29}$, but

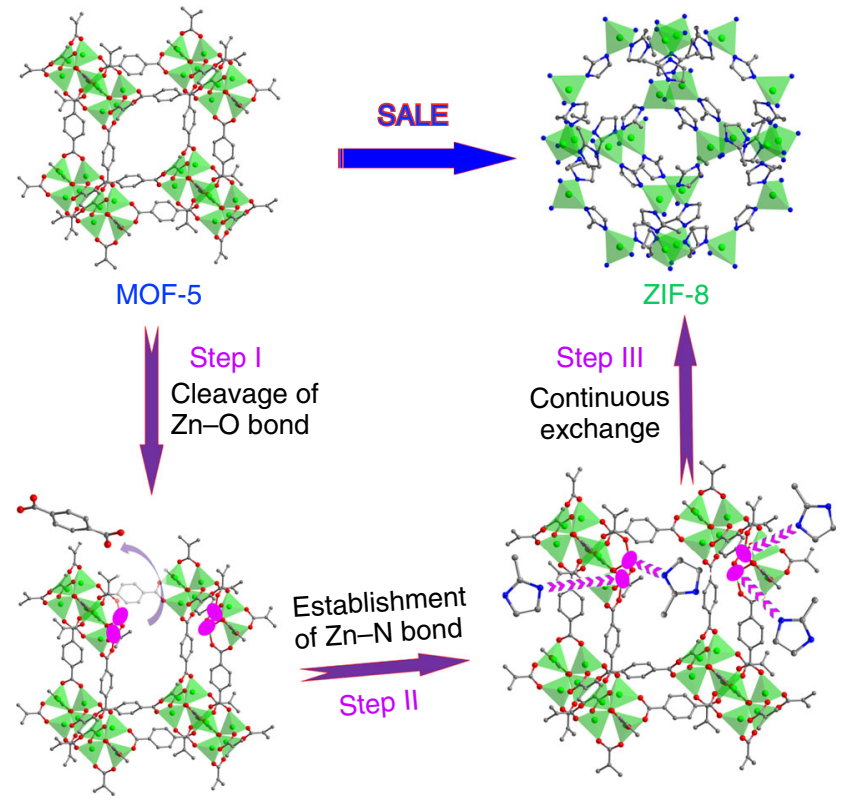

Fig. 1 Schematic representation. A typical solvent-assisted ligand exchange (SALE) process with the transformation from MOF-5 to ZIF-8 as an example (gray ball: $C$, red ball: $O$, blue ball: $N$, green ball: $Z n$ ), the pink elliptic regions denoted unsaturated $\mathrm{Zn}^{2+}$ nodes after the cleavage of $\mathrm{Zn}-\mathrm{O}$ coordination.

the relatively high internal resistance and poor stability truly retards the further promotion of performance. MOF-derived nanoporous carbon demonstrates a feasible option, which can integrate the porous nature and nano-architectures of pristine MOF precursors with excellent conductivity and stability of carbon by a simple carbonization treatment ${ }^{30}$. In view of this, as a potential application of these MOF nanostructures, the as-prepared MOFs are subsequently carbonized to fabricate nanoporous carbon for $\mathrm{Na}^{+}$ion storage. Electrochemical tests indicate that due to the specific architectures inherited from MOF precursors, these nanoporous carbon materials exhibit much better electrochemical energy storage properties than that of common ZIF-8 solid particle-derived carbon, and double-shelled ZnCo bimetallic ZIF nanotube-derived nanoporous carbon delivers very competitive performance compared to previously reported carbon materials. Our present work demonstrates a pioneering study to schematically refine the management of MOF nanostructures. The strategy we propose here can be applied more generally to other MOFs, which offers the strong possibility of realizing a massive selection of MOF nanostructures, hence extending their utilization scope remarkably.

\section{Results}

Synthesis and formation mechanism of 3D MOF architectures. MOF-5, assembled by $\mathrm{Zn}_{4} \mathrm{O}^{6+}$ clusters and $\mathrm{BDC}^{2-}(\mathrm{BDC}=1,4-$ benzenedicarboxylate) ligands, is unstable even under atmospheric conditions because its weak metal-oxygen coordination can be disrupted by water via ligand displacement ${ }^{31}$. The assynthesized MOF-5 had a hexahedral shape and smooth surface (Supplementary Fig. 2a). When MOF-5 was placed in 1.2 M 2methylimidazole (Hmim) ethanol solution at room temperature overnight, the surface of the resulting material became rougher, and hollow features were observed in some broken nanocubes (Supplementary Fig. 2b). The enlarged scanning electron microscopy (SEM) image revealed that such hollow nanocubes possessed a micro-nano superstructure that was composed of many stacked nanoparticles (Supplementary Fig. 2c), implying that the transformation was not controlled by the epitaxial growth 


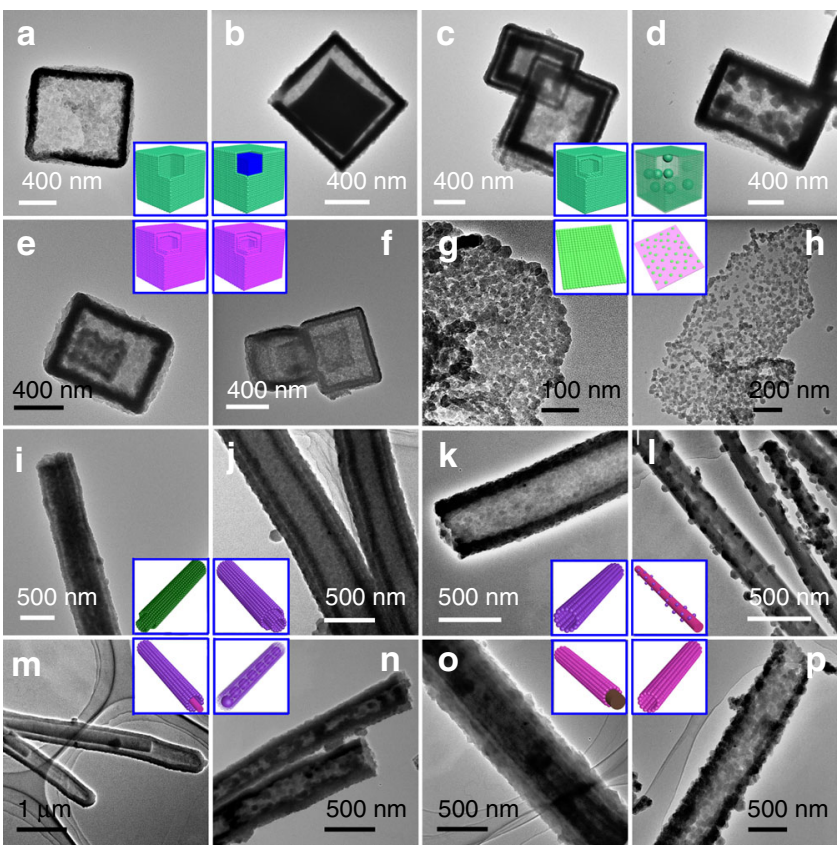

Fig. 2 Zeolitic imidazolate framework-based nanoarchitectures. TEM images of SH-ZIF-8 nanocube (a), yolk-shell MOF-5@ZIF-8 nanocube (b), DH-ZIF-8 nanocube (c), ball-in-box ZIF-8 nanocube (d), DH-ZnCo-ZIF nanocube (e), TH-ZnCo-ZIF nanocube (f), ZIF-8 nanosheet (g), sesame pancake-like Zn-HMT@ZIF-8 nanosheet (h), DT-ZIF-8 nanotube (i), DTZnCo-ZIF nanotube (j), ST-ZnCo-ZIF nanotube (k), bead-on-string hybrid structure (I), nanowire-nanotube hybrid structure $(\mathbf{m})$, peapod-like structure (n),ZnCo-MOF-74@ZnCo-ZIF core-shell structure (o) and ZnCo-MOF-74-derived ZnCo-ZIF nanotube (p).

mechanism. Transmission electron microscopy (TEM) images further confirmed the single-shelled structure (Fig. 2a, Supplementary Fig. 2d), and the corresponding energy dispersive X-ray spectroscopy (EDX) mapping images indicated a uniform element distribution (Supplementary Fig. 2e). The X-ray diffraction (XRD) pattern of the hollow nanocubes matched well with the characteristic diffraction peaks of ZIF-8, and no impurity peak was detected (Supplementary Fig. 2f). Moreover, Fourier transform infrared (FT-IR) spectra clearly clarified the coordination between deprotonated $\mathrm{Hmim}\left(\mathrm{mim}^{-}\right.$) and $\mathrm{Zn}^{2+}$ (Supplementary Fig. 3). Consequently, single-shelled hollow ZIF-8 nanocubes (SH-ZIF-8) were prepared. By simply shortening the time to 2 min, it was easy for us to obtain the MOF-5@ZIF-8 yolk-shell structure (Fig. 2b, Supplementary Fig. 4). If the temperature was increased to $45^{\circ} \mathrm{C}$, MOF-5 evolved to double-shelled hollow ZIF8 (DH-ZIF-8) (Fig. 2c, Supplementary Fig. 5).

The transformation of these MOF-5-derived structures actually results from a diffusion-controlled exchange process ${ }^{28}$. In terms of our previous studies, Hmim solution could induce a weakly acidic environment $\left(\mathrm{Hmim} \rightleftharpoons(1-x) \mathrm{H}^{+}+(1-x) \mathrm{mim}^{-}+x \mathrm{Hmim}\right)$, which breaks ionic bonds such as $\mathrm{Co}-\mathrm{OH}$ and $\mathrm{Zn}-\mathrm{OH}^{32,33}$. Accordingly, one can reason that the weaker $\mathrm{Zn}-\mathrm{O}$ tetrahedral coordination of MOF-5 would be deteriorated once exposed to such an environment, and $\mathrm{Zn}^{2+}$ nodes are in an unsaturated state (Step I in Fig. 1). Meanwhile, deprotonated $\mathrm{mim}^{-}$is prone to coordinating with unsaturated $\mathrm{Zn}^{2+}$ and establishing new more stable $\mathrm{Zn}-\mathrm{N}$ tetrahedral coordination ${ }^{31}$, which completes one exchange cycle between $\mathrm{BDC}^{2-}$ and $\mathrm{mim}^{-}$(Step II in Fig. 1). With continuous ligand exchange, each unit cell finally transforms from MOF-5 to ZIF-8 (Step III in Fig. 1), and ZIF-8 starts to nucleate on the surface of MOF-5 (Supplementary Fig. 6). The ZIF-8 nanocrystals gradually generate with the time, until a thin ZIF-8 layer compactly covers MOF-5 to produce a core-shell hybrid structure, the ion/ molecule transfer is restricted, and the SALE process is diffusioncontrolled (Supplementary Fig. 7). In this stage, only a limited number of $\mathrm{mim}^{-}$can diffuse inward to create a gradually descending molar ratio of $\mathrm{mim}^{-} / \mathrm{Zn}^{2+}$ from outside to inside, the ZIF particle size would increase in order ${ }^{34}$, and thus the thickness from the outer shell to the inner shells increases certainly (Supplementary Fig. 7). On the one hand, even at a very low concentration of $\mathrm{mim}^{-}$, the ligand can readily disrupt the weak coordination bond of MOF-5; on the other hand, the formation of stable ZIF-8 nuclei requires a certain molar ratio of $\mathrm{mim}^{-} / \mathrm{Zn}^{2+, 34}$. As a result, the recrystallization rate of ZIF- 8 is slower than the dissolution rate of MOF-5. The non-equivalent diffusion gives rise to considerable voids near the interfaces of MOF-5/ZIF-8 in a short time, rightly yielding the yolk-shell structure (Fig. 2b, Supplementary Fig. 6, Supplementary Fig. 8).

It is well known that higher temperature leads to wider vibration amplitude of frames and larger spatial change ${ }^{35}$. The increasing temperature facilitates more effective diffusion of $\mathrm{mim}^{-}$ligands for crystallizing secondary ZIF-8 shell evolved from yolk MOF-5, finally DH-ZIF-8 and even the triple-shelled hollow structure (Supplementary Fig. 7e-h) are prepared at $45^{\circ} \mathrm{C}$. At room temperature, the amount of transferred $\mathrm{mim}^{-}$ligands is insufficient to render the crystallization of ZIF- 8 on yolk MOF-5 surface immediately; simultaneously, by reason of the trend of reducing nucleation energy and the relatively higher $\mathrm{mim}^{-} / \mathrm{Zn}^{2+}$ ratio nearby the shell wall than in the center, the growth of ZIF- 8 thus prefers to continue on the inner wall of ZIF- 8 shell, leading to single-shelled hollow structure with thicker wall (Fig. 2a, Supplementary Fig. 7a, b). When lower concentrated Hmim solution is used, the coordination bond of MOF- 5 can be quickly cleaved, while the ZIF-8 nucleation would only happen by the time the $\mathrm{mim}^{-} / \mathrm{Zn}^{2+}$ ratio reaches a certain value; in this case, it is preferable to grow large-sized ZIF- 8 inside the shell, and we consequently obtain an interesting ball-in-box ZIF-8 architecture (Fig. 2d, Supplementary Fig. 6o, Supplementary Fig. 9). If the concentration is further diluted to $0.15 \mathrm{M}$, without adequate $\mathrm{Hmim}$ ligands for the crystallization of ZIF-8, the product is actually an inhomogeneous mixture containing metastable metal-organic coordination compounds ${ }^{36}$, and the transformation from MOF-5 to ZIF-8 is incomplete (Supplementary Fig. 10a-d). In very concentrated solutions, big cracks can be observed on ZIF-8 nanocubes (Supplementary Fig. 10e-h), owing to the stress concentration generated from such violent phase transition. As a consequence, a proper concentration range is needed not only to drive a rational kinetics for crystallizing ZIF-8, but also to offer steady ligand exchange for inheriting the shape of MOF-5 nanocubes; a higher concentration helps with faster kinetics for phase separation to yield yolk-shell structure, and facilitates greater mim $^{-}$concentration gradient along the vertical direction of shell wall afterwards, which is in favor of larger $\mathrm{mim}^{-} / \mathrm{Zn}^{2+}$ ratio inside the hollow interior; the $\mathrm{mim}^{-} / \mathrm{Zn}^{2+}$ ratio finally determines the evolution behavior of MOF-5 core as discussed above (Supplementary Fig. 6), and we obtain SH-ZIF-8 and ball-in-box structure. It is noted that very high $\mathrm{mim}^{-}$concentration might also enable sufficient diffused $\mathrm{mim}^{-}$ligands in the enclosed shell, forming secondary ZIF-8 shell derived from the internal yolk (mentioned below in ZIF-71-derived double-shelled hollow ZIF-8), which is broadly equivalent to the effect of increasing temperature. Furthermore, the dissolution of MOF-5 should keep in step with the recrystallization of ZIF- $8^{37}$, otherwise, if the dissolution process is too fast to provide available substrate for nucleation, taking for example, water as the solvent, it generates monodispersed ZIF-8 particles (Supplementary Fig. 11).

Heterobimetallic MOFs have attracted increasing attention recently ${ }^{12,38}$, we here also prepared $\mathrm{Zn} / \mathrm{Co}$-containing ZIF 
(ZnCo-ZIF) using Co-doped MOF-5 (ZnCo-MOF-5) as the mother MOF. Both $\mathrm{Zn}^{2+}$ and $\mathrm{Co}^{2+}$ ions are known to exist in either tetrahedral or octahedral coordination modes; however, in the presence of weak-field ligands such as carboxylates and solvent molecules, $\mathrm{Zn}^{2+}$ favors tetrahedral coordination, while $\mathrm{Co}^{2+}$ prefers octahedral coordination ${ }^{39}$. Therefore, it is easier to rebuild $\mathrm{Zn}-\mathrm{N}$ tetrahedral coordination (for ZIF-8) than Co-N tetrahedral coordination (for ZIF-67) in a Hmim solution, and theoretically, the recrystallization rate of $\mathrm{ZnCo-MOF-5-derived}$ ZnCo-ZIF is lower than that of MOF-5-derived ZIF-8. In our experiment, if the reaction proceeded at $45^{\circ} \mathrm{C}$, the cubic shape of ZnCo-MOF-5 was lost, and the final products were microspheres with sizes from tens of nanometers to several micrometers (Supplementary Fig. 12). We thus carried out the reaction in a $0{ }^{\circ} \mathrm{C}$ icebox to slow down the reaction kinetics. Single-shelled (Supplementary Fig. 13), double-shelled (Fig. 2e, Supplementary Fig. 14) and triple-shelled (Fig. 2f, Supplementary Fig. 14) hollow ZnCo-ZIF structures could be achieved when carefully manipulating the reaction kinetics similar to MOF-5-derived ZIF-8 hollow structures. Apart from time, temperature, concentration, solvent type and heteroatom, the type of mother MOF inherently affects bond energy and thus the cleavage of coordination bonds. ZIF-8, ZIF-7 and ZIF-71 have similar Zn-N tetrahedral coordination and SOD topology, but their linker $\mathrm{pK}_{\mathrm{a}}$ values follow the trend $\mathrm{pK}_{\mathrm{a}}(\mathrm{ZIF}-8)>\mathrm{pK}_{\mathrm{a}}(\mathrm{ZIF}-7)>\mathrm{pK}_{\mathrm{a}}(\mathrm{ZIF}-71)$. The higher $\mathrm{pK}_{\mathrm{a}}$ is of the linker substituent, the better the stability of the coordination bond ${ }^{31,40,41}$. Our experimental results verify the theoretical expectation that the transformation from ZIF-7 to ZIF-8 requires more severe conditions to overcome the higher reaction barrier in comparison with the cases of ZIF-71 and MOF-5. In addition, ZIF-7-derived single-shelled hollow ZIF-8 (Supplementary Fig. 15), ZIF-71-derived single-shelled (Supplementary Fig. 16) and double-shelled (Supplementary Fig. 17) hollow ZIF-8 are fabricated naturally.

Synthesis of 2D MOF architectures. Based on encouraging results from the nanostructure regulation of $3 \mathrm{D}$ MOFs, we generalize this strategy to $1 \mathrm{D}$ and $2 \mathrm{D}$ MOF nanoarchitectures. $\mathrm{Zn}$ hexamine coordination framework (Zn-HMT) nanosheets are selected as the $2 \mathrm{D}$ mother $\mathrm{MOF}^{42}$. Instead of water, ethanol, N,Ndimethylformamide and $\mathrm{N}$-methyl pyrrolidone, methanol was found to be the best solvent for the successful synthesis of ZIF-8 nanosheets, indicating that solvent type plays a crucial role in the SALE process (Supplementary Fig. 18). Placing Zn-HMT in 0.75 $\mathrm{M}$ and $0.25 \mathrm{M}$ Hmim methanol solutions for $15 \mathrm{~min}, 2 \mathrm{D}$ ZIF-8 nanosheets (Fig. 2g, Supplementary Fig. 19) and sesame pancakelike Zn-HMT@ZIF-8 nanosheets (Fig. 2h, Supplementary Fig. 20) were obtained, respectively. We did not obtain a 2D core-shell nanostructure because of the excessive thinness of the Zn-HMT nanosheets. The thickness of ZIF-8 nanosheets increased compared to Zn-HMT nanosheets, and each ZIF-8 nanosheet exhibited a 2D stacking architecture of ZIF-8 nanaoparticles (Supplementary Fig. 19e). The growth of ZIF-8 nanocrystals first started at the edge of Zn-HMT nanosheets where more unsaturated coordination bonds were exposed (Supplementary Fig. 20ce). As mentioned that the coordination stability of mother MOF influences the ligand exchange. When a very stable $2 \mathrm{D}$ porphyrin paddlewheel framework-3 MOF nanosheet was used as the mother $\mathrm{MOF}^{43}$, it failed to fabricate $\mathrm{ZnCo}$-ZIF nanosheets (Supplementary Fig. 21).

Synthesis of 1D MOF architectures. As the convex surface and small radii of curvature is detrimental for interfacial bonding during subsequent heterogeneous nucleation and growth, reports on diverse and complex 1D MOF nanoarchitectures have seldom been seen so far. We find that the direct transformation from mother MOFs to daughter MOFs can address this issue well. 1D M-BTC $\quad(\mathrm{M}=\mathrm{Co}, \quad \mathrm{Zn} ; \quad \mathrm{BTC}=1,3,5$-benzenetricarboxylate $)$ nanowires were employed to establish 1D ZIF-based nanoarchitectures ${ }^{44}$. In the presence of $1.2 \mathrm{M} \mathrm{Hmim}$ ethanol/water mixed

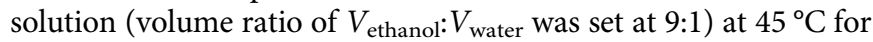
$2 \mathrm{~h}$, Zn-BTC nanowires converted to double-shelled ZIF- 8 nanotubes (DT-ZIF-8, Fig. 2i, Supplementary Fig. 22). The formation of double-shelled nanotube structure was virtually similar to those of 3D ZIF double-shelled hollow structures, the only difference between them was that 1D configuration caused confining diffusion along the axis direction. With the continuous and fast accumulation of vacancies at the ZIF-8/Zn-BTC phase interface by a non-equivalent diffusion effect, phase separation would take place; the initially generated ZIF-8 layer on Zn-BTC nanowire would grow into the outer nanotube, and the inner nanotube finally evolved from the unreacted Zn-BTC nanowire. The very thick inner nanotube wall of each DT-ZIF-8 was identical to the inner shell wall of MOF-5-derived DH-ZIF-8, and the hollow feature of the inner tube was not as obvious. Likewise the involvement of $\mathrm{Co}^{2+}$ created more moderate ligand exchange conditions, which deferred the vacancy clustering and slowed down the ZnCo-ZIF crystallization, resulting in the delaying phase separation. It was interesting to see the thickness of the inner and the outer tube walls for double-shelled ZnCo-ZIF nanotubes (DT-ZnCo-ZIF, Fig. 2j, Supplementary Fig. 23) was almost the same when a bit of $\mathrm{Co}^{2+}$ was introduced (Fig. 2j, Supplementary Fig. 23, Supplementary Fig. 24a, b). With the increasing $\mathrm{Co}^{2+}$ content, the phase separation might not happen; or the ligand transfer was hindered by the thick outer tube wall, the low molar ratio of $\mathrm{mim}^{-} / \mathrm{Zn}^{2+}$ was not able to crystallize the secondary ZIF-8 shell, thus single-shelled ZnCo-ZIF nanotubes (ST-ZnCo-ZIF) were formed (Fig. 2k, Supplementary Fig. 25). We also observed some intriguing exceptions (Supplementary Fig. 24c-f); a triple-shelled trend emerged at the tips of some nanotubes (Supplementary Fig. 24d), and in particular, a few triple-shelled nanotubes were observed (Supplementary Fig. 24ef). By adjusting the reaction time, bead-on-string (Fig. 2l, Supplementary Fig. 26), core-shell (Supplementary Fig. 27) and nanowire-nanotube (Fig. 2m, Supplementary Fig. 28) hybrid structures were prepared. The floccules in the nanowire-nanotube hybrids were considered as the intermediate species of $\mathrm{ZnCo}$-ZIF (Supplementary Fig. 28d). If nanowire-nanotube hybrids reacted with Hmim again, we obtained a peapod-like $\mathrm{ZnCo-ZIF}$ structure (Fig. 2n, Supplementary Fig. 29) rather than DT-ZnCo-ZIF, suggesting a very special environment formed in the confined space of the nanotubes. These prepared 1D double-shelled nanotube, nanowire-nanotube and peapod-like MOF structures are also scarce for inorganic crystals and cannot be fabricated in a traditional way (Supplementary Fig. 30). Similarly, the use of more stable ZnCo-MOF-74 as the mother MOF yields core-shell hybrid (Fig. 2o, Supplementary Fig. 31) and single-shelled nanotube structures (Fig. 2p, Supplementary Fig. 32) which have an obviously grainy surface ${ }^{45}$, and the particle size of $\mathrm{ZnCo}$ ZIF is larger due to the high Co content of the mother MOF, whereas excess Co causes the loss of morphology control (Supplementary Fig. 33).

Fabrication and characterization of nanoporous carbon. Now, we have fabricated 21 different MOF materials and designed 13 different types of MOF architectures which cover most of the configurations that emerge in inorganic crystals; the proposed approach shows big advantages of versatility, simplicity, largeyield product and reproducibility. The synthesis routes of these MOF materials and structures all stem from the same SALE 


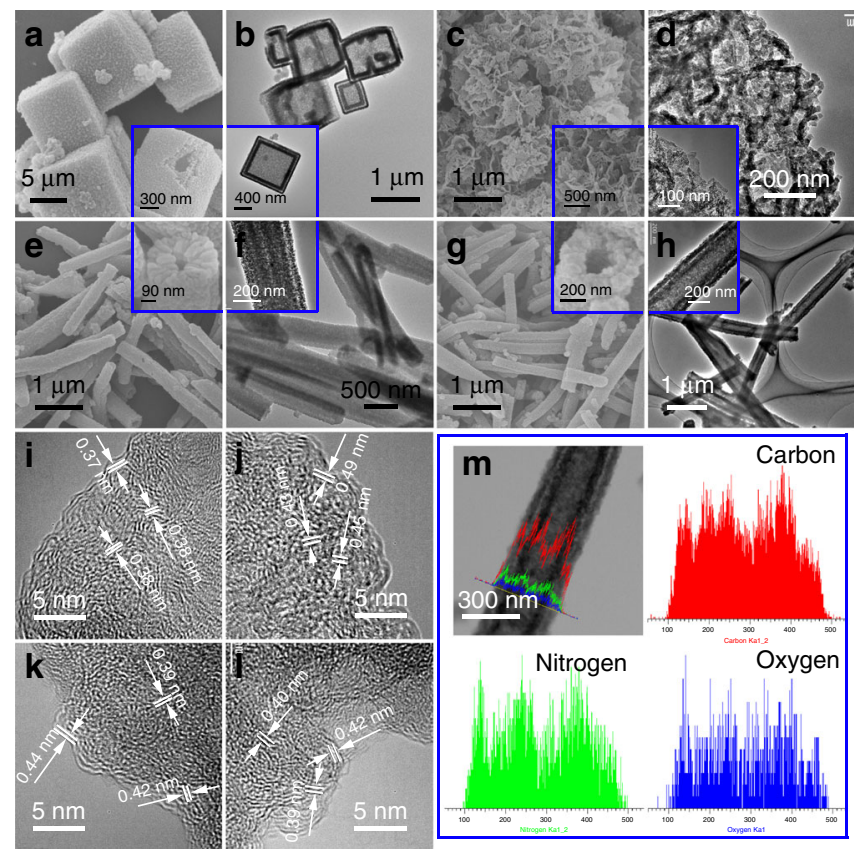

Fig. 3 Zeolitic imidazolate framework-derived nanoporous carbon. SEM (a) and TEM (b) images of 3D DH-Zn-ZIF-C; SEM (c) and TEM (d) images of 2D NS-Zn-ZIF-C; SEM (e) and TEM (f) images of 1D DT-Zn-ZIF-C; SEM $(\mathbf{g})$ and TEM (h) images of $1 D$ DT-ZnCo-ZIF-C; high-resolution TEM images of 3D DH-Zn-ZIF-C (i), 2D NS-Zn-ZIF-C (j), 1D DT-Zn-ZIF-C (k) and 1D DT-ZnCo-ZIF-C (I); EDX linear scanning images of 1D DT-ZnCoZIF-C (m)

strategy, which is a rare and commendable achievement. In this paper, we do not demonstrate the overall application potentials of these MOF architectures but take electrochemical $\mathrm{Na}^{+}$storage as an example. Due to the poor conductivity and instability, the asprepared MOFs are not directly used as the electrode materials. Instead, a carbonization treatment is conducted to obtain MOFderived nanoporous carbon. 3D DH-ZIF-8 nanocubes, 2D ZIF-8 nanosheets, 1D DT-ZIF-8 nanotubes and 1D DT-ZnCo-ZIF nanotubes were carbonized at $900{ }^{\circ} \mathrm{C}$ for $1 \mathrm{~h}$ to prepare the corresponding porous carbon materials, namely, DH-Zn-ZIF-C (Fig. 3a, b), NS-Zn-ZIF-C (Fig. 3c, d), DT-Zn-ZIF-C (Fig. 3e, f) and DT-ZnCo-ZIF-C (Fig. 3g, h), respectively. In addition, a solid ZIF-8 particle-derived porous carbon counterpart (s-Zn-ZIF-C) was prepared for comparison (Supplementary Fig. 34). The porous carbon materials basically maintained the initial morphology of their precursor MOFs, and there was no severe fracture or agglomeration; only distinct shrinkage could be observed. The $\mathrm{C}(002)$ diffraction peaks for DH-Zn-ZIF-C and NS-Zn-ZIF$\mathrm{C}$ were located at the highest and lowest $2 \theta$ angles, respectively (Supplementary Fig. 35). The high-resolution TEM images (Fig. 3i-l) also verified the graphitization degree of four ZIFderived carbon materials; DH-Zn-ZIF-C had the smallest interplanar spacing of $C(002)(0.37-0.38 \mathrm{~nm})$ and the highest graphitization degree in comparison with NS-Zn-ZIF-C (0.43-0.49 $\mathrm{nm})$, DT-Zn-ZIF-C $(0.39-0.44 \mathrm{~nm})$ and DT-ZnCo-ZIF-C $(0.39-0.42 \mathrm{~nm})$. As the previous study demonstrated that a critical minimum spacing of $0.37 \mathrm{~nm}$ was often required for good $\mathrm{Na}^{+}$insertion properties ${ }^{46}$, the enlarged interlayer distance would not only facilitate $\mathrm{Na}^{+}$ion diffusion ${ }^{47}$, but also improve the activation polarization during the $\mathrm{Na}^{+}$intercalation/deintercalation processes. EDX mapping characterization verified the uniform $\mathrm{N}$-doping in these ZIF-derived carbons (Fig. $3 \mathrm{~m}$, Supplementary Fig. 36). The N-doping content of s-Zn-ZIF-C,
DH-Zn-ZIF-C, NS-Zn-ZIF-C, DT-Zn-ZIF-C and DT-ZnCo-ZIF$\mathrm{C}$ was finally determined to be $11.11 \%, 3.69 \%, 13.34 \%, 9.30 \%$ and $8.47 \%$, respectively (Supplementary Fig. 37b-e, Supplementary Table 1) by X-ray photoelectron spectroscopy (XPS). Due to the different electron configuration of $\mathrm{N}$ in the carbon lattices, graphitic $\mathrm{N}$ was inert to $\mathrm{Na}^{+}$; in contrast, both pyridinic $\mathrm{N}$ and pyrrolic $\mathrm{N}$ were very active in capturing $\mathrm{Na}^{+}$ions for electrochemical energy storage, and the induced electrostatic repulsion effect resulted in the expansion of the interlayer distance ${ }^{47}$. Hence, the largest $\mathrm{C}(002)$ interplanar spacing value, observed for NS-Zn-ZIF-C, could be attributed to this material containing the maximum amount of pyrrolic and pyridinic N-doping. The Gband shift in Raman spectra further confirmed the above XRD and XPS analyses (Supplementary Fig. 37f). The specific surface area and pore size distribution were evaluated by the nitrogen sorption technique (Supplementary Fig. 38), and 2D NS-Zn-ZIF$\mathrm{C}$ showed the lowest Brunauer-Emmett-Teller (BET) surface area value of $38.4 \mathrm{~m}^{2} \mathrm{~g}^{-1}$, while $3 \mathrm{D} \mathrm{s}-\mathrm{Zn}$-ZIF-C achieved the highest BET value of $1237.2 \mathrm{~m}^{2} \mathrm{~g}^{-1}$. The Barrett-Joyner-Halenda pore size distribution plots of DH-Zn-ZIF-C and NS-Zn-ZIF-C displayed a trend for pore sizes larger than $10 \mathrm{~nm}$. Both DT-Zn-ZIF$\mathrm{C}$ and DT-ZnCo-ZIF-C showed a relatively narrow pore size distribution $(<10 \mathrm{~nm})$, and most of their average pore sizes ranged from $\sim 1-2 \mathrm{~nm}$, although DT-ZnCo-ZIF-C had more $\sim 2-4$ $\mathrm{nm}$ mesopores. Micropores and mesopores might result from the inheritance of ZIF frameworks, the evaporation of $\mathrm{Zn}$ and the removal of $\mathrm{Co} / \mathrm{Zn}$ species by acid leaching, while the generation of macropores could be originated from the severe shrinkage of ZIF nanoparticle-stacking during carbonization ${ }^{48,49}$. Note that the hierarchical porous architecture significantly affects $\mathrm{Na}^{+}$ storage performance ${ }^{50}$. The micropores in porous carbon can offer more active sites to contribute to $\mathrm{Na}^{+}$adsorption and thus the specific capacity, while the mesoporous and macroporous structures chiefly serve as interconnected pathways for accessible ion transfer and buffer the volume change in the charge-discharge process for good cycling stability.

Electrochemical $\mathrm{Na}^{+}$storage performance. The electrochemical $\mathrm{Na}^{+}$storage properties of ZIF-derived porous carbon were estimated by assembling 2032 type coin cells. Figure 4a shows the cyclic voltammetry (CV) plots of DT-ZnCo-ZIF-C; a broad peak located at $\sim 0.01-1.0 \mathrm{~V}$ can be identified in the $1^{\text {st }}$ cycle, which is attributed to the formation of solid electrolyte interface (SEI) film ${ }^{42}$. The $3^{\text {rd }} \mathrm{CV}$ curve nearly completely overlaps the $2^{\text {nd }}$ cycle, suggesting an excellent reversible $\mathrm{Na}^{+}$storage property of DTZnCo-ZIF-C. The galvanostatic charge-discharge profiles of the DT-ZnCo-ZIF-C electrode were recorded in the potential range of $\sim 0-3 \mathrm{~V}$ at a current density of $0.1 \mathrm{Ag}^{-1}$ (Fig. $4 \mathrm{~b}$ ). The charge and discharge capacities in the first cycle are 465 and $625 \mathrm{mAh} \mathrm{g}^{-1}$, respectively, indicating an initial coulombic efficiency of $74.4 \%$, and the irreversible capacity loss is caused by the formation of the SEI film and the decomposition of electrolyte ${ }^{51}$. DT-ZnCo-ZIF-C delivers the highest specific capacity among all the porous carbon electrodes, and 3D s-Zn-ZIF-C shows the most inferior $\mathrm{Na}^{+}$storage performance (Fig. 4c). At current densities of $0.05,0.1,0.2,0.5,1,2,5$ and $10 \mathrm{~A} \mathrm{~g}^{-1}$, DT-ZnCo-ZIF-C exhibits reversible specific capacities of 474, 455, 432, 396, 364, 305,216 and $178 \mathrm{mAh} \mathrm{g}^{-1}$, respectively. When the current density is returned to $0.1 \mathrm{~A} \mathrm{~g}^{-1}$, an enhanced capacity of $490 \mathrm{mAh}^{-1}$ is achieved; the possible reason for this phenomenon is that electrode activation occurs during the rate cycling so that full penetration of the electrodes is realized to afford more active sites for $\mathrm{Na}^{+}$storage ${ }^{47}$. We also observe that the high-rate performance of DT-Zn-ZIF-C is worse than that of NS-Zn-ZIF-C although DT-Zn-ZIF-C has a higher specific capacity at low 
a

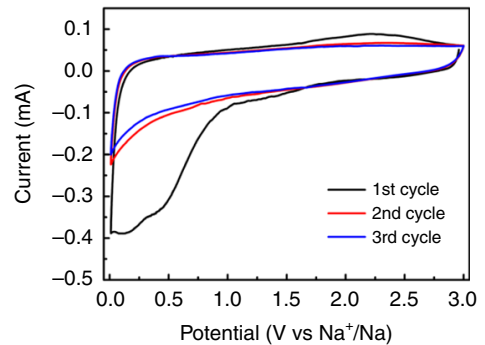

d

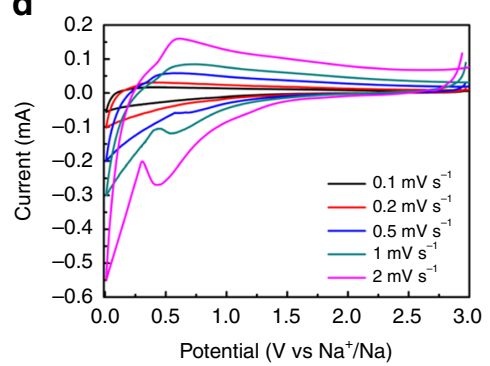

g

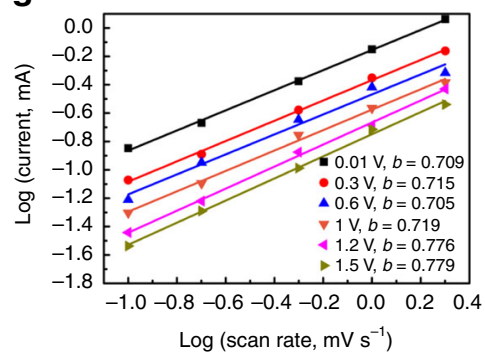

b

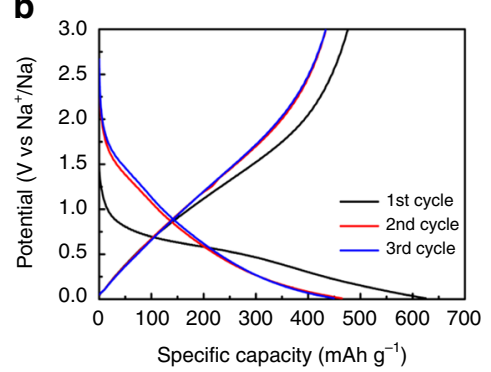

e

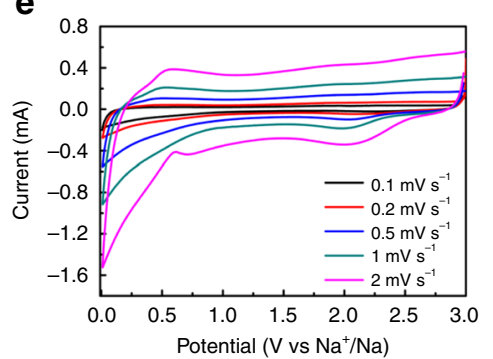

h

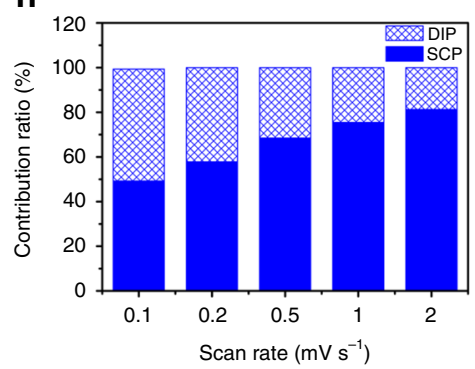

c

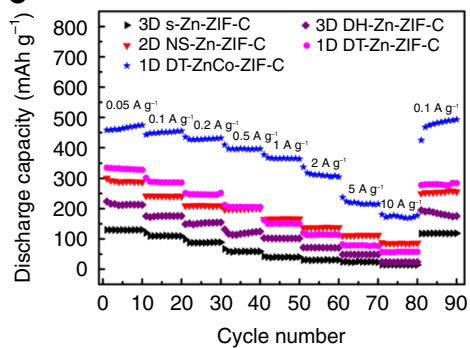

f

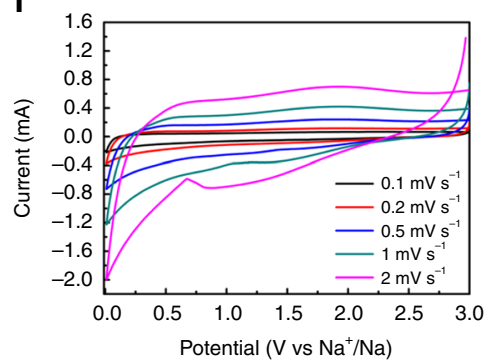

i

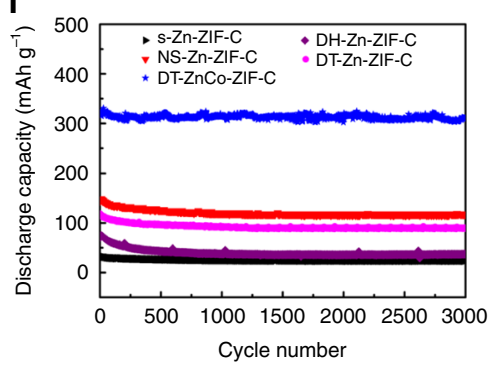

Fig. 4 Electrochemical properties of the prepared nanoporous carbon. $C V$ curves at a scan rate of $0.1 \mathrm{mV} \mathrm{s}^{-1}$ (a) and charge-discharge voltage profiles at a current density of $0.1 \mathrm{Ag}^{-1}$ (b) of DT-ZnCo-ZIF-C; rate capability of 3D s-Zn-ZIF-C, 3D DH-Zn-ZIF-C, 2D NS-Zn-ZIF-C, 1D DT-Zn-ZIF-C and 1D DTZnCo-ZIF-C (c); CV plots of 3D DH-Zn-ZIF-C (d), 2D NS-Zn-ZIF-C (e) and 1D DT-ZnCo-ZIF-C (f) at different scan rates; the linear relationships between the logarithm current and logarithm scan rate $(\mathbf{g})$ and the contribution ratio of DIP and SCP versus scan rate (h) calculated from CV curves of 1D DT-ZnCoZIF-C; long-term cycling performance at a current density of $2 \mathrm{Ag}^{-1}$ (i).

current density; in addition, DH-Zn-ZIF-C manifests only a slightly larger capacity than s-Zn-ZIF-C at $10 \mathrm{~A} \mathrm{~g}^{-1}$; furthermore, $\mathrm{s}-\mathrm{Zn}$-ZIF-C exhibits the most inferior $\mathrm{Na}^{+}$storage properties.

To obtain a better understanding of how the abovementioned different behaviors affect electrochemical $\mathrm{Na}^{+}$storage, $\mathrm{CV}$ curves of all ZIF-derived porous carbon are measured at various scan rates (Fig. 4d-f, Supplementary Fig. 39). According to previous studies, the relationship between current $(i)$ and scan rate $(v)$ can be described by the equation $i=\mathrm{a} v^{b}$, where a and $\mathrm{b}$ are adjustable parameters ${ }^{52}$. The $\mathrm{Na}^{+}$storage behavior is determined by the $b$ value. Generally, when $b=0.5$, the electrochemical storage kinetics are subject to a diffusion-controlled intercalation process (DIP); when $b=1$, the kinetics are controlled by a surfaceinduced capacitive process (SCP). The b values of DT-ZnCo-ZIF$\mathrm{C}$ at $\sim 0-1.5 \mathrm{~V}$ are within the range of $\sim 0.7-0.78$ (Fig. $4 \mathrm{~g}$ ), revealing the combined effect of the battery-type behavior and capacitive effect. Furthermore, the contribution ratio of DIP and SCP to the total capacity can be quantitatively calculated by the equation $i=\mathrm{k}_{1} v+\mathrm{k}_{2} v^{1 / 2}$, in which $i$ represents the current at a certain potential, and $\mathrm{k}_{1} v$ and $\mathrm{k}_{2} v^{1 / 2}$ indicate contributions from DIP and SCP, respectively ${ }^{53}$. The SCP contribution ratio of DTZnCo-ZIF-C increased from $49.3 \%$ to $81.3 \%$ with the increasing scan rate (Fig. 4h). In contrast, capacitive behavior (SCP) dominates the $\mathrm{Na}^{+}$storage process for NS-Zn-ZIF-C, while the major contribution to capacity for s-Zn-ZIF-C, DH-Zn-ZIF-C and DT-Zn-ZIF-C comes from DIP process (Supplementary
Fig. 40), which reasonably explains the inferior rate capacity. In addition to $\mathrm{CV}$ analysis, electrochemical impedance spectroscopy (EIS) is also analyzed. Each Nyquist plot (Supplementary Fig. 41) consists of a semicircle in the high-frequency region and a straight line in the low-frequency region, which respectively represents the charge transfer ability and ion transport efficiency. The larger diameter of the semicircle for 3D s-Zn-ZIF-C and DH$\mathrm{Zn}$-ZIF-C carbon particles corresponds to the higher ohmic resistance relative to $1 \mathrm{D}$ and $2 \mathrm{D}$ carbon nanostructures, and the worse conductivity endows the electrode with higher ohmic polarization. In addition, the steeper slope of the straight line for 2D NS-Zn-ZIF-C and 1D DT-ZnCo-ZIF-C indicates more effective $\mathrm{Na}^{+}$diffusion in comparison with $3 \mathrm{D}$ s-Zn-ZIF-C, 3D $\mathrm{DH}-\mathrm{Zn}$-ZIF-C and 1D DT-Zn-ZIF-C. As is known, during the charge-discharge process, the battery always suffers from battery polarization, which can be divided into ohmic polarization, activation polarization and concentration polarization. Although NS-Zn-ZIF-C had a very low surface area, its excellent conductivity improves the ohmic polarization, and it has the largest interlayer distance of the $\mathrm{C}(002)$ plane, which mitigates the negative influence of activation polarization and concentration polarization, resulting in an extraordinary rate capability. In contrast, s-Zn-ZIF-C has a high surface area and N-doping, but its solid particle structure causes the greatest ohmic resistance and blocks the $\mathrm{Na}^{+}$transport pathway, causing it to exhibit the lowest specific capacity and poor rate capability. All of the ZIF-derived 
nanoporous carbon show better electrochemical properties than the common solid ZIF-8 particle-derived counterpart, suggesting the significance of rational design of MOF architectures. Stemming from the unique double-shelled hollow nanotube structure, DT-ZnCo-ZIF-C takes advantage of a relatively high surface area and $\mathrm{N}$-doping for active sites, a favorable pore distribution for ion diffusion and good conductivity for charge transfer; as a result, the highest specific capacity and superior rate capability could be realized. More importantly, DT-ZnCo-ZIF-C retained $96.6 \%$ of the original value after 3000 repeated cycles at $2 \mathrm{~A} \mathrm{~g}^{-1}$, demonstrating outstanding cycling stability (Fig. 4i). In addition, the as-synthesized DT-ZnCo-ZIF-C also shows better or comparably competitive $\mathrm{Na}^{+}$storage performances compared to other reported MOF-derived carbon as well as other carbon materials (Supplementary Tables 2 and 3) 42,47,51,54-69.

\section{Discussion}

In summary, we have developed a versatile strategy for the design of diverse and complex MOF architectures. By precisely controlling the reaction kinetics to adjust the balance between the cleavage rate of old bonds and the formation rate of new bonds, 21 different MOF materials and 13 different types of MOF configurations ranging from $3 \mathrm{D}$ to $2 \mathrm{D}$ and $1 \mathrm{D}$ structures have been successfully synthesized, covering almost all of the usual inorganic crystal configurations. As a proof-of-concept application, DT-ZnCo-ZIF-C as a $\mathrm{Na}^{+}$ion battery electrode demonstrates remarkable energy storage performance, which can be ascribed to the unique double-shelled nanotube architecture. More importantly, SALE is expected as a powerful strategy to offer unlimited possibilities for the design of other MOFs with various architectures.

\section{Methods}

Synthesis of 3D Zn-MOF-5/ZnCo-MOF-5 nanocubes. In an ice-water bath, $0.1 \mathrm{mmol}$ of 1,4-benzenedicarboxylic acid was dissolved in $15 \mathrm{~mL}$ of N,N-dimethylformamide (DMF), which was dropwise added into $35 \mathrm{~mL}$ of $0.2 \mathrm{M} \mathrm{Zn}$ $\left(\mathrm{CH}_{3} \mathrm{COO}\right)_{2} \mathrm{DMF}$ solution under magnetic stirring for $15 \mathrm{~min}$. Subsequently, the obtained mixture was transferred into a Teflon-lined stainless steel autoclave with a capacity of $50 \mathrm{~mL}$, and the autoclave was placed in a $95^{\circ} \mathrm{C}$ oven for $10 \mathrm{~h}$ and then cooled to room temperature naturally. The resulting white precipitates were washed with DMF several times, collected by centrifugation at 4000 r.p.m. for $10 \mathrm{~min}$, and dried at $80^{\circ} \mathrm{C}$ in a vacuum drying oven overnight. Finally, 3D ZnMOF-5 nanocubes were synthesized. The synthesis of ZnCo-MOF-5 was similar to that of $\mathrm{Zn}-\mathrm{MOF}-5$ except that the molar ratio of $\mathrm{Zn}\left(\mathrm{CH}_{3} \mathrm{COO}\right)_{2} \cdot 2 \mathrm{H}_{2} \mathrm{O} / \mathrm{Co}$ $\left(\mathrm{CH}_{3} \mathrm{COO}\right)_{2} \cdot 4 \mathrm{H}_{2} \mathrm{O}$ for preparing $\mathrm{ZnCo}-\mathrm{MOF}-5$ was $19: 1$, and the prepared $\mathrm{ZnCo}$ MOF-5 had a pink color.

Synthesis of 3D ZIF-7 nanoparticles. First, $2.5 \mathrm{mmol}$ of benzimidazole was dissolved in $50 \mathrm{~mL}$ of methanol, which was then added into $50 \mathrm{~mL}$ of $0.1 \mathrm{mM} \mathrm{Zn}$ $\left(\mathrm{NO}_{3}\right)_{2} \cdot 6 \mathrm{H}_{2} \mathrm{O}$ DMF solution. The mixture was vigorously stirred at room temperature for $6 \mathrm{~h}$. The white ZIF-7 nanoparticles were collected by centrifugation at 4000 r.p.m. for $10 \mathrm{~min}$, washed with ethanol and water for several times, and dried in an $80^{\circ} \mathrm{C}$ oven overnight.

Synthesis of 3D ZIF-71 nanoparticles. First, $3 \mathrm{mmol}$ of 4,5-dichloroimidazole and $1 \mathrm{mmol}$ of $\mathrm{Zn}\left(\mathrm{CH}_{3} \mathrm{COO}\right)_{2} \cdot 2 \mathrm{H}_{2} \mathrm{O}$ were separately dissolved in $15 \mathrm{~mL}$ of methanol, and the two solutions were mixed at room temperature with magnetic stirring for $2 \mathrm{~h}$. The precipitates were centrifuged at 4000 r.p.m. for $10 \mathrm{~min}$, washed with ethanol and water several times, and dried at $80^{\circ} \mathrm{C}$ overnight in an oven. White ZIF-71 nanoparticles were ultimately obtained.

Synthesis of 2D Zn-HMT nanosheets. First, $7 \mathrm{mmol}$ of hexamethylenetetramine (HMT) and $14.25 \mathrm{mmol}$ of $\mathrm{Zn}\left(\mathrm{NO}_{3}\right)_{2} \cdot 6 \mathrm{H}_{2} \mathrm{O}$ were dissolved in $50 \mathrm{~mL}$ and $30 \mathrm{~mL}$ of ethanol solution, respectively. The $\mathrm{Zn}\left(\mathrm{NO}_{3}\right)_{2} \cdot 6 \mathrm{H}_{2} \mathrm{O}$ solution was then added to the HMT solution, and white precipitates were immediately generated. The precipitates were collected by centrifugation at 4000 r.p.m. for $10 \mathrm{~min}$, washed with ethanol several times, and dried in an $80^{\circ} \mathrm{C}$ vacuum oven overnight, yielding $\mathrm{Zn}-\mathrm{HMT}$ nanosheets.

Synthesis of 2D ZnCo-PPF-3 nanosheets. First, $0.015 \mathrm{mmol}$ of $\mathrm{Zn}\left(\mathrm{NO}_{3}\right)_{2} \cdot 6 \mathrm{H}_{2} \mathrm{O}$, $0.135 \mathrm{mmol}$ of $\mathrm{Co}\left(\mathrm{NO}_{3}\right)_{2} \cdot 6 \mathrm{H}_{2} \mathrm{O}, 0.1 \mathrm{mmol}$ of $4,4^{\prime}$-bipyridine and $100 \mathrm{mg}$ of polyvinylpyrrolidone (average mol wt 40,000) were dissolved in $60 \mathrm{~mL}$ of DMFethanol mixed solution (volume ratio of $V_{\mathrm{DMF}}: V_{\text {ethanol }}=3: 1$ ) in a $100 \mathrm{~mL}$ vial. Next, $0.05 \mathrm{mmol}$ of 5,10,15,20-tetrakis(4-carboxylphenyl)porphyrin was dissolved in $20 \mathrm{~mL}$ of DMF-ethanol mixed solution (volume ratio of $V_{\mathrm{DMF}}: V_{\text {ethanol }}=3: 1$ ), which was dropwise added into the former solution and then sonicated for $25 \mathrm{~min}$. Afterwards, the vial was sealed and heated to $80^{\circ} \mathrm{C}$ for $24 \mathrm{~h}$. The resulting red precipitates were collected by centrifugation at 8000 r.p.m. for $15 \mathrm{~min}$, washed with ethanol several times, and dried at $80^{\circ} \mathrm{C}$ in an oven overnight, ultimately yielding ZnCo-PPF-3 nanosheets.

Synthesis of 1D Zn-BTC/ZnCo-BTC nanowires. First, $2.15 \mathrm{mmol}$ of $\mathrm{Zn}$ $\left(\mathrm{CH}_{3} \mathrm{COO}\right)_{2} \cdot 2 \mathrm{H}_{2} \mathrm{O}$ and $2 \mathrm{mmol}$ of $1,3,5$-benzenetricarboxylic acid $\left(\mathrm{H}_{3} \mathrm{BTC}\right)$ were dissolved in $10 \mathrm{~mL}$ and $90 \mathrm{~mL}$ of water, respectively. The former solution was added into the latter solution at $100{ }^{\circ} \mathrm{C}$ with magnetic stirring for $5 \mathrm{~min}$. The white precipitates were centrifuged at 3000 r.p.m. for $10 \mathrm{~min}$, washed with ethanol several times, and dried at $80^{\circ} \mathrm{C}$ in an oven overnight, yielding Zn-BTC nanowires. The synthesis of pink-colored $\mathrm{ZnCo-BTC}$ nanowires was similar to that of $\mathrm{Zn}$-BTC nanowires except that the molar ratio of $\mathrm{Zn}\left(\mathrm{CH}_{3} \mathrm{COO}\right)_{2} \cdot 2 \mathrm{H}_{2} \mathrm{O} / \mathrm{Co}$ $\left(\mathrm{CH}_{3} \mathrm{COO}\right)_{2} \cdot 4 \mathrm{H}_{2} \mathrm{O}$ for preparing $\mathrm{ZnCo}-\mathrm{BTC}$ was varied. XZnYCo-BTC denoted $\mathrm{ZnCo}-\mathrm{BTC}$ with different molar ratios of $\mathrm{Zn} / \mathrm{Co}$, in which $\mathrm{X}$ and $\mathrm{Y}$ corresponded to the molar percentages of $\mathrm{Zn}$ and Co, respectively.

Synthesis of 1D ZnCo-MOF-74 nanowires. First, $1 \mathrm{mmol}$ of $\mathrm{Zn}$ $\left(\mathrm{CH}_{3} \mathrm{COO}\right)_{2} \cdot 2 \mathrm{H}_{2} \mathrm{O}$ and $3 \mathrm{mmol}$ of $\mathrm{Co}\left(\mathrm{CH}_{3} \mathrm{COO}\right)_{2} \cdot 4 \mathrm{H}_{2} \mathrm{O}$ were dissolved in $10 \mathrm{~mL}$ of water, which was then added into $90 \mathrm{~mL}$ of 2,5-dihydroxy-1,4-benzenedicarboxylic acid $(2 \mathrm{mmol})$ aqueous solution heated at $100{ }^{\circ} \mathrm{C}$ in an oil bath for $40 \mathrm{~min}$. The earth yellow ZnCo-MOF-74 was centrifuged at 1000 r.p.m. for $10 \mathrm{~min}$, washed with ethanol several times, and dried at $80^{\circ} \mathrm{C}$ in an oven overnight. $\mathrm{XZnYCo-MOF-74} \mathrm{denoted} \mathrm{ZnCo-MOF-74} \mathrm{with} \mathrm{different} \mathrm{molar} \mathrm{ratios} \mathrm{of} \mathrm{Zn} / \mathrm{Co}$, in which $\mathrm{X}$ and $\mathrm{Y}$ corresponded to the molar percentages of $\mathrm{Zn}$ and Co, respectively.

Synthesis of Zn-MOF-5-derived ZIF-8-based nanostructures. When the prepared $\mathrm{Zn}$-MOF-5 powder was placed in $1.2 \mathrm{M} 2$-methylimidazole (Hmim) ethanol solution at room temperature overnight, the transformation from Zn-MOF-5 nanocubes to single-shelled hollow ZIF-8 nanocubes (SH-ZIF-8) occurred. When the reaction time was decreased to 2 min, MOF-5@ZIF-8 yolk-shelled nanocubes could be obtained. When the reaction was conducted at $45^{\circ} \mathrm{C}$ overnight, doubleshelled hollow ZIF-8 nanocubes (DH-ZIF-8) were synthesized. In addition, if the concentration of Hmim ethanol solution was diluted from $1.2 \mathrm{M}$ to $0.6 \mathrm{M}$ during the reaction, an interesting ball-in-box ZIF- 8 architecture was consequently produced. All of the products were collected by centrifugation at 3000 r.p.m. for $15 \mathrm{~min}$, washed with ethanol and water several times, and dried in a $70{ }^{\circ} \mathrm{C}$ oven.

Synthesis of ZnCo-MOF-5-derived ZnCo-ZIF nanostructures. When the assynthesized $\mathrm{ZnCo-MOF-5}$ powder was added into $0.6 \mathrm{M} \mathrm{Hmim}$ ethanol solution for $6 \mathrm{~h}$ in a $0{ }^{\circ} \mathrm{C}$ icebox, single-shelled $\mathrm{ZnCo}$-ZIF hollow nanocubes were synthesized. If the concentration of Hmim ethanol solution was increased to $1.2 \mathrm{M}$, double-shelled $\mathrm{ZnCo}$-ZIF hollow nanocubes were prepared, and some tripleshelled ZnCo-ZIF hollow nanocubes could also be observed in the product. The resulting ZnCo-ZIF nanostructures were collected by centrifugation at 3000 r.p.m. for $15 \mathrm{~min}$, washed with ethanol and water several times, and dried in a $70{ }^{\circ} \mathrm{C}$ oven.

Synthesis of ZIF-7-/ZIF-71-derived ZIF-8 hollow particles. When the prepared ZIF-7 was added into $2.4 \mathrm{M} \mathrm{Hmim}$ ethanol solution at $45^{\circ} \mathrm{C}$ for $6 \mathrm{~h}$, single-shelled ZIF-8 hollow nanoparticles could be prepared. Under the same conditions, doubleshelled ZIF-8 hollow nanoparticles would be realized when the mother MOF was ZIF-71; if 1.2 M Hmim ethanol solution was used, single-shelled ZIF-8 hollow nanoparticles were obtained. The resulting ZIF-8 hollow nanoparticles were collected by centrifugation at 3000 r.p.m. for $15 \mathrm{~min}$, washed with ethanol and water several times, and dried in a $70^{\circ} \mathrm{C}$ oven.

Synthesis of Zn-HMT-derived ZIF-8-based nanostructures. When the prepared $\mathrm{Zn}$-HMT was added to $0.75 \mathrm{M}$ and $0.2 \mathrm{M} \mathrm{Hmim}$ methanol solution at $30^{\circ} \mathrm{C}$ for 15 min, 2D ZIF-8 nanosheets and sesame pancake-like Zn-HMT@ZIF-8 nanosheets could be synthesized, respectively. The products were collected by centrifugation at 3000 r.p.m. for $15 \mathrm{~min}$, washed with ethanol and water several times, and dried in a $70^{\circ} \mathrm{C}$ oven.

Synthesis of Zn-BTC-derived double-shelled ZIF-8 nanotubes. When the asprepared Zn-BTC nanowires were transferred into $1.2 \mathrm{M} \mathrm{Hmim}$ ethanol/water mixed solution (volume ratio of $V_{\text {ethanol: }} V_{\text {water }}=9: 1$ ) at $45^{\circ} \mathrm{C}$ for $2 \mathrm{~h}$, doubleshelled ZIF-8 nanotubes (DT-ZIF-8) were synthesized. Under the same reaction conditions, when 90Zn10Co-BTC nanowires and 50Zn50Co-BTC nanowires were used as the mother MOFs, they gave rise to double-shelled ZnCo-ZIF nanotubes (DT-ZnCo-ZIF) and single-shelled ZnCo-ZIF nanotubes (ST-ZnCo-ZIF), respectively. 
Synthesis of ZnCo-BTC-derived ZIF-based nanostructures. The preparation of $\mathrm{ZnCo}-\mathrm{BTC}$-derived $\mathrm{ZnCo}-\mathrm{ZIF}$-based nanostructures was similar to that of $\mathrm{Zn}$ BTC-derived double-shelled ZIF-8 nanotubes, only 90Zn10Co-BTC nanowire was used as the mother MOF. When $90 \mathrm{Zn} 10 \mathrm{Co}-\mathrm{BTC}$ nanowires were transferred into $1.2 \mathrm{M} \mathrm{Hmim}$ ethanol/water mixed solution (volume ratio of $V_{\text {ethanol }} V_{\text {water }}=9: 1$ ) at $45^{\circ} \mathrm{C}$ for $10 \mathrm{~min}, 5 \mathrm{~min}$ and $1 \mathrm{~min}, 1 \mathrm{D}$ 90Zn10Co-BTC@ZnCo-ZIF nanowirenanotube structures, 1D 90Zn10Co-BTC@ZnCo-ZIF core-shell nanowires and 1D 90Zn10Co-BTC@ZnCo-ZIF bead-on-string structures were produced, respectively. When placing the 90Zn10Co-BTC@ZnCo-ZIF nanowire-nanotube structure into $1.2 \mathrm{M} \mathrm{Hmim}$ ethanol/water mixed solution $45^{\circ} \mathrm{C}$ for $2 \mathrm{~h}$ again, a peapod-like $\mathrm{ZnCo}$-ZIF structure emerged. The products were all collected by centrifugation at 3000 r.p.m. for $15 \mathrm{~min}$, washed with ethanol and water several times, and dried in a $70{ }^{\circ} \mathrm{C}$ oven.

\section{Synthesis of ZnCo-MOF-74-derived ZIF-based nanostructures. The} 20Zn80Co-MOF-74 nanowires were selected as the mother MOF. Compared to $\mathrm{ZnCo}-\mathrm{BTC}$, the 20Zn80Co-MOF-74 nanowires were placed in $1.2 \mathrm{M} \mathrm{Hmim}$ ethanol/water mixed solution (volume ratio of $V_{\text {ethanol: }}: V_{\text {water }}=5: 5$ ) to induce the transformation. When the reaction continued for $2 \mathrm{~h}$, single-shelled ZnCo-ZIF nanotubes were synthesized. When the reaction was shortened to $15 \mathrm{~min}$, the resulting material evolved to a 20Zn80Co-MOF-74@ZnCo-ZIF core-shell structure. All of the products were collected by centrifugation at 1000 r. p. m. for $10 \mathrm{~min}$, washed with ethanol and water several times, and dried in a $70^{\circ} \mathrm{C}$ oven.

Synthesis of solid ZIF-8 particles. For comparison, $0.8 \mathrm{mmol}$ of $\mathrm{Zn}$ $\left(\mathrm{CH}_{3} \mathrm{COO}\right)_{2} \cdot 2 \mathrm{H}_{2} \mathrm{O}$ and $0.3 \mathrm{~g}$ of polyvinylpyrrolidone (average mol wt 30,000$)$ were dissolved in $20 \mathrm{~mL}$ of methanol and placed in a refrigerator for $1 \mathrm{~h}$. In the meantime, $3.2 \mathrm{mmol}$ of Hmim was dissolved in $20 \mathrm{~mL}$ of methanol and placed in the refrigerator for $1 \mathrm{~h}$. Subsequently, the former solution was dropwise added to the latter solution at room temperature with magnetic stirring for $24 \mathrm{~h}$. Solid ZIF-8 particles were collected by centrifugation, washed with ethanol and water several times, and dried in a $70^{\circ} \mathrm{C}$ oven.

Synthesis of ZIF-derived nanoporous carbon nanostructures. The MOF precursors, including solid ZIF-8 particles, MOF-5-derived DH-ZIF-8 nanocubes, ZnHMT-derived ZIF-8 nanosheets, Zn-BTC-derived DT-ZIF-8 nanotubes and ZnCoBTC-derived $\mathrm{ZnCo}$-ZIF nanotubes, were heated to $900^{\circ} \mathrm{C}$ in an $\mathrm{Ar}$ atmosphere for $2 \mathrm{~h}$ at a heating rate of $2{ }^{\circ} \mathrm{C} / \mathrm{min}$. After cooling to room temperature naturally, the black powders were washed with $5 \mathrm{M} \mathrm{HNO}_{3}$ aqueous solution at $80^{\circ} \mathrm{C}$ for $24 \mathrm{~h}$ to remove the $\mathrm{Zn}$ or Co species. The resulting materials were collected by centrifugation, washed with ethanol and water several times, and finally dried in a vacuum oven at $120^{\circ} \mathrm{C}$

Material characterizations. The X-ray diffraction (XRD) patterns were recorded by a Rigaku D/MAX2500VL/PC X-ray diffractometer with $\mathrm{Cu} \mathrm{Ka}$ radiation. The morphology and microstructure of the samples were examined by field-emission scanning electron microscopy (SEM, SU8020) and transmission electron microscopy (TEM, JEM-2100). Nitrogen adsorption-desorption analysis was carried out by using a Micromeritics ASAP 2020 instrument at $77 \mathrm{~K}$, and the surface area values were calculated by the Brunauer-Emmett-Teller (BET) method in the relative pressure $\left(P / P_{\mathrm{o}}\right)$ range of $0.002-1.0$. X-ray photoelectron spectroscopy (XPS) was performed by a Thermo Fisher X-ray photoelectron spectrometer (ESCALAB250), and the results were fitted using XPSpeak software according to the principle of minimum residual standard deviation. The Raman spectrum was collected from an Ar laser (Renishaw inVia) with a $532 \mathrm{~nm}$ laser excitation source at room temperature. The Fourier transform infrared spectrum (FT-IR) was collected on a Perkin Elmer Fourier-Transform infrared spectrometer by using the $\mathrm{KBr}$ wafer technique.

Electrochemical measurements. The electrochemical properties of ZIF-derived nanoporous carbon nanostructures were estimated by assembling 2032 type coin cells. To prepare the working electrodes, the active materials were mixed with Super $\mathrm{P}^{\oplus}$ carbon black and polyvinylidene fluoride at a weight ratio of 80:10:10 in $\mathrm{N}$-methyl pyrrolidinone (NMP) to make a slurry mixture, which were pasted on copper foil and dried in a vacuum oven at $60^{\circ} \mathrm{C}$ for $12 \mathrm{~h}$ and then $120^{\circ} \mathrm{C}$ for another $2 \mathrm{~h}$. The mass loading of the working electrodes was $0.8 \sim 1.0 \mathrm{mg} / \mathrm{cm}^{2}$. Metal $\mathrm{Na}$ foil was used as the counter electrode, and borosilicate glass microfiber (GF/D, Whatman) was used as the separator. The electrolyte was $1 \mathrm{M} \mathrm{NaClO}$ in a mixed solution of ethylene carbonate (EC) and dimethyl carbonate (DMC) (volume ratio of $\left.V_{\mathrm{EC}}: V_{\mathrm{DMC}}=1: 1\right)$. The coin cells were assembled in an Ar-filled glovebox (MIKROUNA) and allowed to rest overnight before the electrochemical tests. The galvanostatic discharge/charge profiles were recorded on a Land CT2001A battery tester (China) in a $25^{\circ} \mathrm{C}$ thermostat (FYL-YS-280L, Beijing Fu Italian Electric, China). The cyclic voltammetry analysis and electrochemical impedance spectroscopy (EIS) measurements were conducted on a CHI660C electrochemical workstation (Chenhua Instrument Company, China). EIS spectra were recorded in a frequency range of $0.1 \mathrm{~Hz} \sim 100 \mathrm{kHz}$ at the open-circuit potential with an ac perturbation of $0.1 \mathrm{~V}$.

\section{Data availability}

The authors declare all data supporting the findings of this study are available within the paper and the Supplementary Information file, or available from the corresponding authors on reasonable request. The source data underlying all figures are provided as a Source Data file.

Received: 28 August 2019; Accepted: 28 November 2019; Published online: 17 February 2020

\section{References}

1. Furukawa, H., Cordova, K. E., O’Keeffe, M. \& Yaghi, O. M. The chemistry and applications of metal-organic frameworks. Science 341, 1230444 (2013).

2. Inokuma, $\mathrm{Y}$. et al. X-ray analysis on the nanogram to microgram scale using porous complexes. Nature 495, 461-466 (2013).

3. Li, L. et al. Ethane/ethylene separation in a metal-organic framework with iron-peroxo sites. Science 362, 443-446 (2018).

4. Trickett, C. A. et al. Identification of the strong Bronsted acid site in a metalorganic framework solid acid catalyst. Nat. Chem. 11, 170-176 (2019).

5. Sheberla, D. et al. Conductive MOF electrodes for stable supercapacitors with high areal capacitance. Nat. Mater. 16, 220-224 (2017).

6. Feng, D. et al. Robust and conductive two-dimensional metal-organic frameworks with exceptionally high volumetric and areal capacitance. Nat. Energy 3, 30-36 (2018).

7. Fang, Z., Bueken, B., Vos, D. E. D. \& Fischer, R. A. Defect-engineered metalorganic frameworks. Angew. Chem. Int. Ed. 54, 7234-7254 (2015).

8. Ma, X. et al. Zeolitic imidazolate framework membranes made by ligandinduced permselectivation. Science 361, 1008-1011 (2018).

9. Ameloot, R. et al. Interfacial synthesis of hollow metal-organic framework capsules demonstrating selective permeability. Nat. Chem. 3, 382-387 (2011).

10. Xu, X., Zhang, Z. \& Wang, X. Well-defined metal-organic-framework hollow nanostructures for catalytic reactions involving gases. Adv. Mater. 27, 5365-5371 (2015)

11. Zhao, M. et al. Metal-organic frameworks as selectivity regulators for hydrogenation reactions. Nature 539, 76-80 (2016).

12. Ahn, S. H., Klein, M. J. \& Manthiram, A. 1D Co- and N-doped hierarchically porous carbon nanotubes derived from bimetallic metal organic framework for efficient oxygen and tri-iodide reduction reactions. Adv. Energy Mater. 7 1601979 (2017)

13. Liu, W. et al. Multi-shelled hollow metal-organic frameworks. Angew. Chem Int. Ed. 56, 1-6 (2017).

14. Shen, K. et al. Ordered macro-microporous metal-organic framework single crystals. Science 359, 206-210 (2018).

15. Zhan, G. \& Zeng, H. C. Hydrogen spillover through matryoshka-type (ZIFs@) $)_{n-1}$ ZIFs nanocubes. Nat. Commun. 9, 3778 (2018).

16. Li, T., Sullivan, J. E. \& Rosi, N. L. Design and preparation of a core-shell metal-organic framework for selective $\mathrm{CO}_{2}$ capture. J. Am. Chem. Soc. 135, 9984-9987 (2013)

17. Zhuang, J. et al. Surfactant-mediated conformal overgrowth of core-shell metal-organic framework materials with mismatched topologies. Small 11, 5551-5555 (2015)

18. $\mathrm{Gu}, \mathrm{Y}$. et al. Controllable modular growth of hierarchical MOF-on-MOF architectures. Angew. Chem. Int. Ed. 56, 15658-15662 (2017).

19. Pang, X., He, Y., Jung, J. \& Lin, Z. 1D nanocrystals with precisely controlled dimensions, compositions, and architectures. Science 353, 1268-1272 (2016).

20. Fenton, J. L., Steimle, B. C. \& Schaak, R. E. Tunable intraparticle frameworks for creating complex heterostructured nanoparticle libraries. Science $\mathbf{3 6 0}$, 513-517 (2018)

21. Ikigaki, K. et al. MOF-on-MOF: oriented growth of multiple layered thin films of metal-organic frameworks. Angew. Chem. Int. Ed. 58, 6886-6890 (2019)

22. Hwang, J., Heil, T., Antonietti, M. \& Schmidt, B. V. K. J. Morphogenesis of metal-organic mesocrystals mediated by double hydrophilic block copolymers. J. Am. Chem. Soc. 140, 2947-2956 (2018)

23. Dissegna, S., Epp, K., Heinz, W. R., Kieslich, G. \& Fischer, R. A. Defective metal-organic frameworks. Adv. Mater. 30, 1704501 (2018).

24. Park, J., Feng, D. \& Zhou, H. C. Structure-assisted functional anchor implantation in robust metal-organic frameworks with ultralarge pores. J. Am. Chem. Soc. 137, 1663-1672 (2015).

25. Deria, P. et al. Beyond post-synthesis modification: evolution of metal-organic frameworks via building block replacement. Chem. Soc. Rev. 43, 5896-5912 (2014).

26. Cui, P., Wang, P., Zhao, Y. \& Sun, W.-Y. Fabrication of desired metal-organic frameworks via post-synthetic exchange and sequential linker installation. Cryst. Growth Des. 19, 1454-1470 (2019). 
27. Meng, W. et al. New mechanistic insight into stepwise metal-center exchange in a metal-organic framework based on asymmetric $\mathrm{Zn}_{4}$ clusters. Chem. Eur. J. 20, 2945-2952 (2014).

28. Khlobystov, A. N. et al. Anion exchange in co-ordination polymers: a solid-state or a solvent-mediated process? CrystEngComm 4, 426-431 (2002).

29. Xu, Y., Li, Q., Xue, H. \& Pang, H. Metal-organic frameworks for direct electrochemical applications. Coord. Chem. Rev. 376, 292-318 (2018).

30. Yang, W., Li, X., Li, Y., Zhu, R. \& Pang, H. Applications of metal-organicframework-derived carbon materials. Adv. Mater. 31, 1804740 (2019).

31. Low, J. J. et al. Virtual high throughput screening confirmed experimentally: porous coordination polymer hydration. J. Am. Chem. Soc. 131, 15834-15842 (2009).

32. $\mathrm{Yu}, \mathrm{D}$. et al. Decorating nanoporous ZIF-67-derived $\mathrm{NiCo}_{2} \mathrm{O}_{4}$ shells on a $\mathrm{Co}_{3} \mathrm{O}_{4}$ nanowire array core for batterytype electrodes with enhanced energy storage performance. J. Mater. Chem. A 4, 10878-10884 (2016).

33. Yu, D. et al. A general route to the synthesis of layer-by-layer structured metal organic framework/graphene oxide hybrid films for high-performance supercapacitor electrodes. J. Mater. Chem. A 5, 16865-16872 (2017).

34. Pan, Y., Liu, Y., Zeng, G., Zhao, L. \& Lai, Z. Rapid synthesis of zeolitic imidazolate framework-8 (ZIF-8) nanocrystals in an aqueous system. Chem. Commun. 47, 2071-2073 (2011).

35. Shao, Z. et al. Modulation of magnetic behavior and $\mathrm{Hg}^{2+}$ removal by solventassisted linker exchange based on a water-stable 3D MOF. Chem. Mater. 30, 7979-7987 (2018).

36. Xu, X. et al. Synthesis of ZIF-8 hollow spheres via MOF-to-MOF conversion. ChemistrySelect 1, 1763-1767 (2016).

37. Mao, D., Wan, J., Wang, J. \& Wang, D. Sequential templating approach: a groundbreaking strategy to create hollow multishelled structures. Adv. Mater. 31, 1802874 (2018)

38. Zhao, S. et al. Ultrathin metal-organic framework nanosheets for electrocatalytic oxygen evolution. Nat. Energy 1, 16184 (2016).

39. Caskey, S. R. \& Matzger, A. J. Selective metal substitution for the preparation of heterobimetallic microporous coordination polymers. Inorg. Chem. 47, 7942-7944 (2008).

40. Bhattacharyya, S. et al. Acid gas stability of zeolitic imidazolate frameworks: generalized kinetic and thermodynamic characteristics. Chem. Mater. 30, 4089-4101 (2018).

41. Colombo, V. et al. High thermal and chemical stability in pyrazolate-bridged metal-organic frameworks with exposed metal sites. Chem. Sci. 2, 1311-1319 (2011).

42. Liu, S., Zhou, J. \& Song, H. 2D Zn-hexamine coordination frameworks and their derived N-rich porous carbon nanosheets for ultrafast sodium storage. Adv. Energy Mater. 8, 1800569 (2018).

43. Cao, F. et al. Synthesis of two-dimensional $\mathrm{CoS}_{1.097} /$ nitrogen-doped carbon nanocomposites using metal-organic framework nanosheets as precursors for supercapacitor application. J. Am. Chem. Soc. 138, 6924-6927 (2016).

44. Yaghi, O. M., Li, H. \& Groy, T. L. Construction of porous solids from hydrogen-bonded metal complexes of 1,3,5-benzenetricarboxylic acid. J. Am. Chem. Soc. 118, 9096-9101 (1996).

45. Rosi, N. L. et al. Rod packings and metal-organic frameworks constructed from rod-shaped secondary building units. J. Am. Chem. Soc. 127, 1504-1518 (2005).

46. Cao, Y. et al. Sodium ion insertion in hollow carbon nanowires for battery applications. Nano Lett. 12, 3783-3787 (2012).

47. Liu, J. et al. Graphitic carbon nitride $\left(\mathrm{g}-\mathrm{C}_{3} \mathrm{~N}_{4}\right)$-derived $\mathrm{N}$-rich graphene with tuneable interlayer distance as a high-rate anode for sodium-ion batteries. Adv. Mater. 31, 1901261 (2019).

48. Wei, J. et al. Graphene-directed assembly route to hierarchically porous Co$\mathrm{N}_{\mathrm{x}}-\mathrm{C}$ catalysts for high-performance oxygen reduction. J. Mater. Chem. A 3, 16867-16873 (2015)

49. Zhong, H. X. et al. ZIF-8 derived graphene-based nitrogen-doped porous carbon sheets as highly efficient and durable oxygen reduction electrocatalysts. Angew. Chem. Int. Ed. 53, 1-7 (2014).

50. Kim, J. et al. Rational Design of carbon nanomaterials for electrochemical sodium storage and capture. Adv. Mater. 31, 1803444 (2019).

51. Chen, Y. et al. Nitrogen-doped carbon for sodium-ion battery anode by selfetching and graphitization of bimetallic MOF-based composite. Chem 3, 152-163 (2017).

52. Lindström, H. et al. $\mathrm{Li}^{+}$ion insertion in $\mathrm{TiO}_{2}$ (Anatase). 2. voltammetry on nanoporous films. J. Phys. Chem. B 101, 7717-7722 (1997).

53. Liu, T. C., Pell, W. G., Conway, B. E. \& Roberson, S. L. Behavior of molybdenum nitrides as materials for electrochemical capacitors comparison with ruthenium oxide. J. Electrochem. Soc. 145, 1882-1888 (1998).

54. Zhang, W. et al. Hollow carbon nanobubbles: monocrystalline MOF nanobubbles and their pyrolysis. Chem. Sci. 8, 3538-3546 (2017).
55. Zou, G. et al. 3D hollow porous carbon microspheres derived from Mn-MOFs and their electrochemical behavior for sodium storage. J. Mater. Chem. A 5 23550-23558 (2017)

56. Liu, S., Zhou, J. \& Song, H. Tailoring highly N-doped carbon materials from hexamine-based MOFs: superior performance and new insight into the roles of N configurations in Na-ion storage. Small 14, e1703548 (2018).

57. $\mathrm{Du}, \mathrm{M}$. et al. Stereoselectively assembled metal-organic framework (MOF) host for catalytic synthesis of carbon hybrids for alkaline-metal-ion batteries. Angew. Chem. Int. Ed. 58, 5307-5311 (2019).

58. Kong, L., Zhu, J., Shuang, W. \& Bu, X.-H. Nitrogen-doped wrinkled carbon foils derived from MOF nanosheets for superior sodium storage. Adv. Energy Mater. 8, 1801515 (2018).

59. Mahmood, A. et al. Ultrafast sodium/potassium-ion intercalation into hierarchically porous thin carbon shells. Adv. Mater. 31, e1805430 (2019).

60. Wen, Y. et al. Expanded graphite as superior anode for sodium-ion batteries. Nat. Commun. 5, 4033 (2014)

61. Hou, H., Banks, C. E., Jing, M., Zhang, Y. \& Ji, X. Carbon quantum dots and their derivative $3 \mathrm{D}$ porous carbon frameworks for sodium-ion batteries with ultralong cycle life. Adv. Mater. 27, 7861-7866 (2015).

62. Wang, S. et al. Free-standing nitrogen-doped carbon nanofiber films: integrated electrodes for sodium-ion batteries with ultralong cycle life and superior rate capability. Adv. Energy Mater. 6, 1502217 (2016).

63. Lu, P., Sun, Y., Xiang, H., Liang, X. \& Yu, Y. 3D amorphous carbon with controlled porous and disordered structures as a high-rate anode material for sodium-ion batteries. Adv. Energy Mater. 8, 1702434 (2018).

64. Huang, S. et al. N-doping and defective nanographitic domain coupled hard carbon nanoshells for high performance lithium/sodium storage. Adv. Funct. Mater. 28, 1706294 (2018).

65. Quan, B. et al. Solvothermal-derived S-doped graphene as an anode material for sodium-ion batteries. Adv. Sci. 5, 1700880 (2018).

66. Zou, G. et al. Advanced hierarchical vesicular carbon Co-doped with S, P, N for high-rate sodium storage. Adv. Sci. 5, 1800241 (2018).

67. $\mathrm{Hu}, \mathrm{X}$. et al. Nitrogen-rich hierarchically porous carbon as a high-rate anode material with ultra-stable cyclability and high capacity for capacitive sodiumion batteries. Nano Energy 56, 828-839 (2019).

68. Yao, X. et al. Defect-rich soft carbon porous nanosheets for fast and high capacity sodium-ion storage. Adv. Energy Mater. 9, 1803260 (2018).

69. Zou, G. et al. Cube-shaped porous carbon derived from MOF-5 as advanced material for sodium-ion batteries. Electrochim. Acta 196, 413-421 (2016).

\section{Acknowledgements}

We acknowledge the funding support from the National Natural Science Foundation of China (Nos. 21606217, U1810204 and 91534203), Fundamental Research Funds for the Central Universities (No. PA2019GDQT0015) and the 111 Project "New Materials and Technology for Clean Energy" (B18018). J.W.C. would also like to thank the financial support from the China Scholarship Council during his visit to Prof. Pulickel M. Ajayan's group at Rice University.

\section{Author Contributions}

D.B.Y., J.W.C., T.W.X. and Y.C.W. conceived the project. D.B.Y., Q.S., Q.J.S. and Y.L.Z planned and performed the experiments and collected and analyzed the data. B.W., L.G., Y.W., Y.Z., Y.Q.Q., H.T.W. and P.M.A. assisted with the experiments and characterizations. D.B.Y., J.W.C., T.W.X., R.V. and Y.C.W. co-wrote the manuscript. All authors discussed the results and commented on the manuscript. D.B.Y. and Q.S. contributed equally to this work

\section{Competing interests}

The authors declare no competing interests.

\section{Additional information}

Supplementary information is available for this paper at https://doi.org/10.1038/s41467 020-14671-9.

Correspondence and requests for materials should be addressed to J.C., P.M.A., T.X. or Y.W.

Peer review information Nature Communications thank the anonymous reviewers fo their contributions to the peer review of this work. Peer review reports are available.

Reprints and permission information is available at http://www.nature.com/reprints

Publisher's note Springer Nature remains neutral with regard to jurisdictional claims in published maps and institutional affiliations. 
(c) (i) Open Access This article is licensed under a Creative Commons Attribution 4.0 International License, which permits use, sharing, adaptation, distribution and reproduction in any medium or format, as long as you give appropriate credit to the original author(s) and the source, provide a link to the Creative Commons license, and indicate if changes were made. The images or other third party material in this article are included in the article's Creative Commons license, unless indicated otherwise in a credit line to the material. If material is not included in the article's Creative Commons license and your intended use is not permitted by statutory regulation or exceeds the permitted use, you will need to obtain permission directly from the copyright holder. To view a copy of this license, visit http://creativecommons.org/ licenses/by/4.0/.

(C) The Author(s) 2020 